\title{
New Riesz representations of linear maps associated with certain boundary value problems and their applications
}

\author{
Wei Yang ${ }^{1}$, Jiannan Duan ${ }^{1}$, Wenmin $\mathrm{Hu}^{2}$ and Jing Zhang ${ }^{3 *}$
}

\section{"Correspondence:}

zhangj8901@foxmail.com

${ }^{3}$ Faculty of Science and Technology,

Tapee College, Surathani, 84000,

Thailand

Full list of author information is

available at the end of the article

\section{Abstract}

In this paper, we obtain new Riesz representations of continu linear maps associated with certain boundary value problems in t' set of à ' 'osed bounded convex non-empty subsets of any Banach space. A- app ᄏtions, the Riesz integral representation results are also given.

Keywords: Riesz decomposition method; 1 res zing vector-valued map

\section{Introduction}

Physicists have long been us' ig so- 'led singular functions such as the Dirac delta function $\delta$, although these carnot tion theory. The Dirar telia funct $n$ in $\delta(x-\xi)$ is equal to zero everywhere except at $\xi$, where it is infinite, and it inte lis ne. According to the classical definition of a function and an integral thes onditio, , are inconsistent. In elementary particle physics, one found the need to evalua ${ }^{2}$ when calculating the transition rates of certain particle interactions [1 In [2], a definition of a product of distributions was given using delta sequences. Howev $\delta^{2}$ as product of $\delta$ with itself, was shown not to exist. In [3], Bremermann $u$ a d the Caucny representations of distributions with compact support to define $\sqrt{\delta_{+}}$and $\log \delta$ iortunately, his definition did not carry over to $\sqrt{\delta}$ and $\log \delta$. In 1964, Gel'fand and Shilov [4] defined $\delta^{[(k)]}(P)$ for an infinitely differentiable function $P\left(x_{1}, x_{2}, \ldots, x_{n}\right)$ such the $P=0$ hypersurface has no singular points, where

$$
P=P\left(x_{1}, x_{2}, \ldots, x_{n}\right)=x_{1}^{2}+x_{2}^{2}+\cdots+x_{p}^{2}-x_{p+1}^{2}-\cdots-x_{p+q}^{2},
$$

$p+q=n$ is the dimension of the Euclidean space $\mathbb{R}^{n}$, the $P=0$ hypersurface is a hypercone with a singular point (the vertex) at the origin. Then they also defined the generalized functions $\delta_{1}^{(k)}(P)$ and $\delta_{2}^{(k)}(P)$ as in the cases $p, q>1$ and $p, q=1$, respectively. To establish the numerous properties of $P$ defined by (1.1) Bliedtner and Hansen first showed that it was a quotient of the larger Feller compactification in [5]. It then turned out that functions that were exactly the uniform limits on compact sets of sequences of bounded harmonic functions allowed a nice integral representation on $P$. They called them continuous linear maps. In developing their properties, Ikegami gave several equivalent conditions that

(c) The Author(s) 2017. This article is distributed under the terms of the Creative Commons Attribution 4.0 International License (http://creativecommons.org/licenses/by/4.0/), which permits unrestricted use, distribution, and reproduction in any medium, provided you give appropriate credit to the original author(s) and the source, provide a link to the Creative Commons license, and indicate if changes were made. 
force them to have an integral representation even with respect to minimal representing measures onthe boundary of $P$ in [6]. Several examples given by the Laplace equation and the heat equation showed that $P$ was in general different from the Martin compactification; It was, however, the same for ordinary harmonic functions on Lipschitz domains. Conditions were also presented that force all positive harmonic functions to be sturdy, extending the results first presented in [5]. Based on earlier work of the authors in [7], and [8] concerning the boundary behavior of continuous linear maps, the second author and Weizsäcker had shown that a required condition was naturally satisfied when the unde lying measure space was second countable. Samuelsson [9] studied the residue of the eneralized function $G^{\lambda}$, where $\lambda$ was a complex number. This generalized function $G^{\lambda}$ have been used for various purposes by several authors; notably for instance the exr iicit of of the duality theorem for a complete intersection in [10], explicit versions of the ndamental principle in [11], sharp approximation by polynomials [9], and estir. ss of soly ons to the Bezout equation in [12]; for further examples in [13] and the referenc therein. One can also use such generalized functions to obtain sharp estim ates $t$ the boundary, such as $H^{p}$-estimates, of explicit solutions to division problems in [1. reira [14] studied the asymptotic behavior in time of the olutions / a coupled system of linear Maxwell equations with thermal effects. The Riesz Las s $s_{\mathrm{r}} \mathrm{Jperty}$ and the stability of a damped Euler-Bernoulli beam with nonuniform thickne. s or density have been studied in [15], where the authors applied a linear br unda. ontrol force in position and velocity at the free end of the beam. Recently, Yan [16, udie $d$ the generalization of distributional product of Dirac's delta in a hypercor w whose re, its are a generalization of formulas that appear in [3]. Furthermore, he al us a $\mathrm{mu}$ ch simpler method of deriving the product $f(r) \cdot \delta^{(k)}(r-1)$ for all non-neg at integer, and $r=\left(x_{1}^{2}+x_{2}^{2}+\cdots+x_{n}^{2}\right)^{1 / 2}$, and then studied a more general product $f(r) \cdot \delta^{(k)}(, \quad$ where $H$ is a regular hypersurface. And they found the product $P^{n} \cdot \delta^{(k)}(P)$ is well as a general product $f(P) \cdot \delta^{(k)}(P)$, where $f$ is a $C^{\infty}$-function on $\mathbb{R}$. Another study c he products of particular distributions and the development of other work can $\mathrm{L}$ ound in $[8,17]$.

By using augmentea, iesz decomposition methods developed by Wang, Huang and Yamini 17], he pu pose of this paper is to study the product $G^{l} \cdot \delta^{(k)}(G)$ and then study a more $\varepsilon_{\text {en }}$. oduct of $f(G) \cdot \delta^{(k)}(G)$, where $f$ is a $C^{\infty}$-function on $\mathbb{R}$ and $\delta^{(k)}(G)$ is the

I. ac delta nction with $k$-derivatives. Meanwhile, we shall show that we can control the $L^{\infty}$, m by the $H^{1}$ norm and a stronger norm with a logarithmic growth or double logarithmic growth. The inequality is sharp for the double logarithmic growth. The result th re is used earlier in our paper to obtain a boundary limit theorem for sturdy harmonic functions and continuous linear maps. Before proceeding to our main results, the following definitions and concepts are required.

\section{Preliminaries}

Definition 2.1 Let $x=\left(x_{1}, x_{2}, \ldots, x_{n}\right)$ be a point of $n$-dimensional Euclidean space $\mathbb{R}^{n}$ and $m$ be a positive integer. The hypersurface $G=G(m, x)$ is defined by

$$
G=G(m, x)=\left(\sum_{i=1}^{p} x_{i}^{2}\right)^{m}-\left(\sum_{j=p+1}^{p+q} x_{j}^{2}\right)^{m},
$$


where $p+q=n$ is the dimension of $\mathbb{R}^{n}$. The hypersurface $G$ is due to Berndtsson and Passare [11]. We observe that putting $m=1$ in (2.1), we obtain

$$
G=G(1, x)=\sum_{i=1}^{p} x_{i}^{2}-\sum_{j=p+1}^{p+q} x_{j}^{2}=P(x)=P
$$

where the quadratic form $P$ is due to Gel'fand and Shilov [4] and is given by (1.1). The hypersurface $G=0$ is a generalization of a hypercone $P=0$ with a singular point ( $\mathrm{t}^{2}$ vertex) at the origin.

Definition 2.2 Let $\operatorname{grad} G \neq 0$, which means there is no singular point on $G=0$. en we define

$$
\left\langle\delta^{(k)}(G), \phi\right\rangle=\int \delta^{(k)}(G) \phi(x) d x
$$

where $\delta^{(k)}$ is the Dirac delta function with $k$-derivatives, $\phi$ is a testmg function in the Schwartz space $S, x=\left(x_{1}, x_{2}, \ldots, x_{n}\right) \in \mathbb{R}^{n}$ and $d x=d_{n} \quad x_{n} d x_{n}$. , M a sufficiently small neighborhood $U$ of any point $\left(x_{1}, x_{2}, \ldots, x_{n}\right)$ of the hypersurface $G=0$, we can introduce a new coordinate system such that $G=0$ becomes one of th coordinate hypersurface. For this purpose, we write $G=u_{1}$ and choose th rema. $\lg u_{i}$ coordinates (with $i=2,3, \ldots, n$ ) for which the Jacobian

$$
D\left(\begin{array}{l}
x \\
u
\end{array}\right)>0
$$

where

$$
D\left(\begin{array}{l}
x \\
u
\end{array}\right)=\frac{\partial\left(x_{1}, x_{2}, .\right.}{\partial\left(\ldots u_{n}\right)}
$$

Thus 2.0 , an be written as

$$
\left\langle\delta^{(k)}(G), \phi\right\rangle=(-1)^{k} \int\left[\frac{\partial^{k}}{\partial G^{k}}\left\{\phi D\left(\begin{array}{l}
x \\
u
\end{array}\right)\right\}\right]_{G=0} d u_{2} d u_{3} \cdots d u_{n} .
$$

The proof of the following lemma is given in [17].

\section{Lemma 2.1 Given the hypersurface}

$$
G=\left(\sum_{i=1}^{p} x_{i}^{2}\right)^{m}-\left(\sum_{j=p+1}^{p+q} x_{j}^{2}\right)^{m}
$$

where $p+q=n$ is the dimension of $\mathbb{R}^{n}$, and $m$ is a positive integer. If we transform to bipolar coordinates defined by

$$
x_{1}=r \omega_{1}, \quad \ldots, \quad x_{p}=r \omega_{p}, \quad x_{p+1}=s \omega_{p+1}, \quad \ldots, \quad x_{p+q}=s \omega_{p+q},
$$


where

$$
\sum_{i=1}^{p} \omega_{i}^{2}=1
$$

and

$$
\sum_{j=p+1}^{p+q} \omega_{j}^{2}=1
$$

Then the hypersurface $G$ can be written by

$$
G=r^{2 m}-s^{2 m},
$$

and we obtain

$$
\left\langle\delta^{(k)}(G), \phi\right\rangle=\int_{0}^{\infty}\left[\left(\frac{1}{2 m s^{2 m-1}} \frac{\partial}{\partial s}\right)^{k}\left\{s^{q-2 m} \frac{\psi(r, s)}{2 m}\right\}\right]_{s} r^{p-1} d r
$$

or

$$
\left.\left\langle\delta^{(k)}(G), \phi\right\rangle=(-1)^{k} \int_{0}^{\infty}\left[\left(\frac{1}{2 m r^{2 m-1}} \frac{\partial}{r}\right)^{p-2 m} \frac{(r, s)}{2 m}\right\}\right]_{r=s} s^{q-1} d s,
$$

where

$$
\psi(r, s)=\int \phi d \Omega^{(p)} d \Omega^{(q,}
$$

and $d \Omega^{(p)}$ and $d \Omega^{(q)}$ c. the elements of surface area on the unit sphere in $\mathbb{R}^{p}$ and $\mathbb{R}^{q}$, respectively.

Now, ye. ume hat $\phi$ vanishes in the neighborhood of the origin, so that these integrals will co, re ny $k$. Now for

$$
(\eta-1)+(q-2 m) \geq 2 m k
$$

$$
k<\frac{1}{2 m}(p+q-2 m)
$$

the integrals in (2.5) converge for any $\phi(x) \in S$. Similarly, for

$$
(q-1)+(p-2 m) \geq 2 m k
$$

or

$$
k<\frac{1}{2 m}(p+q-2 m)
$$


the integrals in (2.6) also converge for any $\phi(x) \in S$. Thus we take (2.5) and (2.6) to be the defining equation for $\delta^{(k)}(G)$. On the other hand, if

$$
k \geq \frac{1}{2 m}(p+q-2 m)
$$

then we shall define $\left\langle\delta_{1}^{*}(G), \phi\right\rangle$ and $\left\langle\delta_{2}^{*}(G), \phi\right\rangle$ as the regularization of (2.5) and (2.6), respectively. For $p>1$ and $q>1$, the generalized function $\delta_{1}^{*(k)}(G)$ and $\delta_{2}^{*(k)}(G)$ are defined by

$$
\left\langle\delta_{1}^{*(k)}(G), \phi\right\rangle=\int_{0}^{\infty}\left[\left(\frac{1}{2 m s^{2 m-1}} \frac{\partial}{\partial s}\right)^{k}\left\{s^{q-2 m} \frac{\psi(r, s)}{2 m}\right\}\right]_{s=r} r^{p-1} d r
$$

for all

$$
k \geq \frac{1}{2 m}(p+q-2 m)
$$

we have

$$
\left\langle\delta_{2}^{*(k)}(G), \phi\right\rangle=(-1)^{k} \int_{0}^{\infty}\left[( \frac { 1 } { 2 m r ^ { 2 m - 1 } } \frac { \partial } { \partial r } ) ^ { k } \left\{r^{p-2 m} \frac{\psi(1}{2 m} \gamma_{r, s} c^{q * 1} d s\right.\right.
$$

for

$$
k \geq \frac{1}{2 m}(p+q-2 m)
$$

In particular, for $m=1, \delta_{1}^{*(k)}$, is redu to $\delta_{1}^{(k)}(G)$, and $\delta_{2}^{*(k)}(G)$ is reduced to $\delta_{2}^{(k)}(G)$ (see [4, p.250]).

\section{Main results}

Assume that both $p>1$, $a>1$. Let

$$
G(x)=G\left(x_{1}, \cdots, \ldots, \hat{x}_{n}\right)=\left(x_{1}^{2}+x_{2}^{2}+\cdots+x_{p}^{2}\right)^{m}-\left(x_{p+1}^{2}+\cdots+x_{p+q}^{2}\right)^{m},
$$

with $p+\ldots$ n the $G=0$ hypersurface is a hypercone with a singular point (the vertex) the origs.

V. tart by assuming that $\phi(x)$ vanishes in a neighborhood of the origin. The distribution $\delta,(G)$ is defined by

$$
\left\langle\delta^{(k)}(G), \phi\right\rangle=(-1)^{k} \int\left[\frac{\partial^{k}}{\partial G^{k}}\left\{\frac{1}{2 m}\left(r^{2 m}-G\right)^{\frac{q}{2 m}-1} \phi\right\}\right]_{G=0} r^{p-1} d r d \Omega^{(p)} d \Omega^{(q)},
$$

which is convergent.

Furthermore, if we transform from $G$ to

$$
s=\left(r^{2 m}-G\right)^{\frac{1}{2 m}},
$$

then we note that

$$
\frac{\partial}{\partial G}=-\left(2 m s^{2 m-1}\right)^{-1} \frac{\partial}{\partial s} .
$$


We may write this in the form

$$
\left\langle\delta^{(k)}(G), \phi\right\rangle=\int\left[\left(\frac{1}{2 m s^{2 m-1}} \frac{\partial}{\partial s}\right)^{k}\left\{s^{q-2 m} \frac{\phi}{2 m}\right\}\right]_{s=r} r^{p-1} d r d \Omega^{(p)} d \Omega^{(q)}
$$

Let us now define

$$
\psi(r, s)=\int \phi d \Omega^{(p)} d \Omega^{(q)}
$$

Hence

$$
\left\langle\delta^{(k)}(G), \phi\right\rangle=\int_{0}^{\infty}\left[\left(\frac{1}{2 m s^{2 m-1}} \frac{\partial}{\partial s}\right)^{k}\left\{s^{q-2 m} \frac{\psi(r, s)}{2 m}\right\}\right]_{s=r} r^{p-1} d r
$$

See Lemma 2.1 for more details.

Theorem 3.1 The product of $G^{l}$ and $\delta^{(k)}(G)$ exists and

$$
G^{l} \cdot \delta^{(k)}(G)= \begin{cases}(-1)^{l} \frac{k !}{k-l} \delta^{k-l}(G) & \text { if } k \geq l \\ 0 & \text { if } k \geq l\end{cases}
$$

Proof From (3.1), we start with

$$
\begin{aligned}
& \left\langle G^{l} \cdot \delta^{(k)}(G), \phi\right\rangle=(-1)^{k} \int_{j}\left[\partial^{k}-\left\{G^{l} \frac{\partial^{m}}{2 m}\left(r^{2 m}-G\right)^{\frac{q}{2 m}-1} \phi\right\}\right]_{G=0} r^{p-1} d r d \Omega^{(p)} d \Omega^{(q)} \\
& =\infty^{\infty}\left[\left(\frac{1}{2 m} \frac{\partial}{s^{2 m-1}} \frac{\partial}{\partial s}\right)^{k}\left\{\left(r^{2 m}-s^{2 m}\right)^{l} s^{q-2 m} \frac{\psi(r, s)}{2 m}\right\}\right]_{s=r} r^{p-1} d r .
\end{aligned}
$$

Making the substr $n s=r^{2 m}, v=s^{2 m}$ and putting $\psi(r, s)=\psi_{1}(u, v)$, we have

$$
\left\{G \quad \left\langle(u,, \varphi)=\frac{1}{4 m^{2}} \int_{0}^{\infty}\left[\left(\frac{\partial}{\partial v}\right)^{k}\left\{(u-v)^{l} v^{\frac{q}{2 m}-1} \psi_{1}(u, v)\right\}\right]_{u=v} u^{\frac{p}{2 m}-1} d u\right.\right. \text {. }
$$

Clea

$$
\begin{aligned}
\left.\frac{\partial^{k}}{\partial v^{k}}\left\{(u-v)^{l} v^{\frac{q}{2 m}-1} \psi_{1}(u, v)\right\}\right|_{u-v}= & \left.\sum_{i=0}^{k}\left(\begin{array}{c}
k \\
i
\end{array}\right) D_{v}^{i}(u-v)^{l} D_{v}^{k-i}\left\{v^{\frac{q}{2 m}-1} \psi_{1}(u, v)\right\}\right|_{u-v} \\
= & \left.\sum^{i<l}\left(\begin{array}{c}
k \\
i
\end{array}\right) D_{v}^{i}(u-v)^{l} D_{v}^{k-i}\left\{v^{\frac{q}{2 m}-1} \psi_{1}(u, v)\right\}\right|_{u-v} \\
& +\left.\left(\begin{array}{c}
k \\
l
\end{array}\right) D_{v}^{i}(u-v)^{l} D_{v}^{k-i}\left\{v^{\frac{q}{2 m}-1} \psi_{1}(u, v)\right\}\right|_{u-v} \\
& +\left.\sum^{i>l}\left(\begin{array}{c}
k \\
i
\end{array}\right) D_{v}^{i}(u-v)^{l} D_{v}^{k-i}\left\{v^{\frac{q}{2 m}-1} \psi_{1}(u, v)\right\}\right|_{u-v} \\
= & I_{1}+I_{2}+I_{3},
\end{aligned}
$$


where

$$
D_{v}^{i}=\partial / \partial v^{i} .
$$

It follows that

$$
I_{1}=I_{3}=0
$$

since $i \neq l$. As for $I_{2}$, we obtain

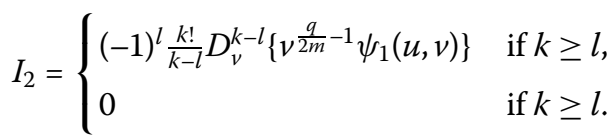

Substituting $I_{2}$ back and using (3.1), we obtain

$$
G^{l} \cdot \delta^{(k)}(G)= \begin{cases}(-1)^{l} \frac{k !}{k-l} \delta^{k-l}(G) & \text { if } k \geq l, \\ 0 & \text { if } k \geq l,\end{cases}
$$

which completes the proof of theorem.

Example 3.1 By letting $m=n=p=1$ in (2.1) an $k=3$ in (3.4), we have

$$
x^{6} \cdot \delta^{\prime \prime \prime}\left(x^{2}\right)=-6 \delta\left(x^{2}\right) \text {. }
$$

Obviously, we can extend Theorem 3.1 $\mathrm{r}$ ore general product as follows.

Theorem 3.2 Letf be a C -functu $n \mathbb{R}$. Then the product of $f(G)$ and $\delta^{(k)}(G)$ exists and

$$
f(G) \delta^{(k)}(G)=\sum_{n}^{k}(t,=(1))^{i} f^{(i)}(0) \delta^{(k-i)}(G) .
$$

Proof Le $=f(G)$ and use Theorem 3.1. Moreover, note that

$$
\begin{aligned}
\left.\frac{\partial^{k}}{\partial v^{k}}\{f,-v) v^{\frac{q}{2 m}-1} \psi_{1}(u, v)\right\}\left.\right|_{u-v} & =\left.\sum_{i=0}^{k}\left(\begin{array}{c}
k \\
i
\end{array}\right) D_{v}^{i} f(u-v) D_{v}^{k-i}\left\{v^{\frac{q}{2 m}-1} \psi_{1}(u, v)\right\}\right|_{u-v} \\
& =\left.\sum_{i=0}^{k}\left(\begin{array}{c}
k \\
i
\end{array}\right)(-1)^{i} f^{(i)}(0) D_{v}^{k-i}\left\{v^{\frac{q}{2 m}-1} \psi_{1}(u, v)\right\}\right|_{u-v} .
\end{aligned}
$$

In particular, we have

$$
\sin G \cdot \delta^{(k)}(G)=\sum_{i=0}^{k}\left(\begin{array}{c}
k \\
i
\end{array}\right)(-1)^{i} \sin \frac{i \pi}{2} \delta^{(k-i)}(G)
$$

and

$$
e^{G} \cdot \delta^{(k)}(G)=\sum_{i=0}^{k}\left(\begin{array}{l}
k \\
i
\end{array}\right)(-1)^{i} \delta^{(k-i)}(G) .
$$


Example 3.2 By letting $m=n=p=1$ in (2.1) and $k=3$ in (3.5), we have

$$
\sin x^{2} \cdot \delta^{\prime \prime \prime}\left(x^{2}\right)=-3 \delta^{\prime \prime}\left(x^{2}\right)+\delta\left(x^{2}\right)
$$

Similarly, by letting $m=n=p=1$ in (2.1) and $k=4$ in (3.6), we have

$$
e^{x^{2}} \cdot \delta^{(4)}\left(x^{2}\right)=\delta^{(4)}\left(x^{2}\right)-4 \delta^{\prime \prime \prime}\left(x^{2}\right)+6 \delta^{\prime \prime}\left(x^{2}\right)-4 \delta^{\prime}\left(x^{2}\right)+\delta\left(x^{2}\right) .
$$

\section{Numerical simulations}

In this section, we give the bifurcation diagrams, phase portraits of model (2.1) to onfirm the above theoretic analysis and show the new interesting complex dynamical beh iors by using numerical simulations. The bifurcation parameters are considered in foln. two cases:

In model (2.1) we choose $\mu=0.3, N=0.7, \beta=1.9, \gamma=0.1, h \in[, 2$. $\quad$ and the initial value $\left(S_{0}, I_{0}\right)=(0.01,0.01)$. We see that model $(2.1)$ has only on sitive ey allibrium $E_{2}$. By calculation we have

$$
\begin{aligned}
& E_{2}\left(S^{*}, I^{*}\right)=E_{2}(0.1474,0.4145), \quad \alpha_{1}=-0.9524, \\
& \alpha_{2}=0.8811, \quad h=\frac{570-4 \sqrt{2,306}}{180},
\end{aligned}
$$

and

$$
(\mu, N, \beta, h, \gamma) \in M_{1}
$$

which shows the correctnd. of Th $2 \mathrm{~m}$ 3.1. From Theorem 3.2, we see that the equilibrium $E_{2}(0.1474,0.4145$ is stable for

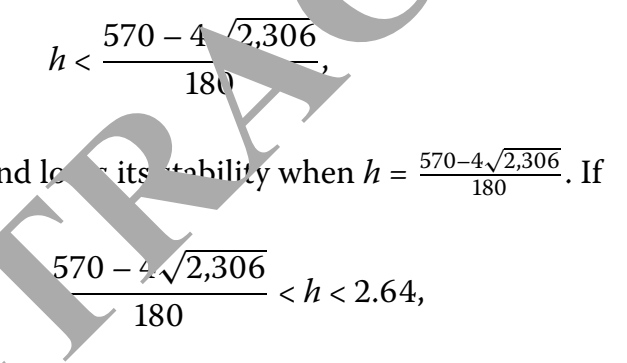

th $n$ there exist period- 2 orbits. Moreover, period- 4 orbits, period- 8 orbits and period16 orbits appear in the rang $h \in[2.65,2.85)$. At last, the $2^{n}$ period orbits disappear and the dynamical behaviors are from non-period orbits to the chaotic set with the increasing of $h$. We also can find that the range $h$ is decreasing with the doubled increasing of the period orbits which indicates the Feigenbaum constant $\delta$. The dynamical behavior processes from period-one orbit to chaos sets show self-similar characteristics. Further, the period-doubling transition leads to the chaos sets as May and Odter obtained in [3].

\section{Conclusions}

In this paper, we firstly obtained the representation of continuous linear maps in the set of all closed bounded convex non-empty subsets of any Banach space. As applications, 
we secondly deduced the Riesz integral representation results for set-valued maps, for vector-valued maps of Diestel-Uhl and for scalar-valued maps of Dunford-Schwartz. Finally, we gave the bifurcation diagrams, phase portraits of related models to confirm the above theoretic analysis and showed the new interesting complex dynamical behaviors by using numerical simulations.

\section{Acknowledgements}

The authors thank the referees for their valuable comments, which greatly improved their paper. This work was supported by the National Natural Science Foundation of China (Grant No. 41171176) and Postgraduate Technology Innovation Project of Hunan Province (Grant No. CX2015B243).

\section{Competing interests}

The authors declare that they have no competing interests.

Authors' contributions

JZ drafted the manuscript. WY helped to prepare the revised manuscript and JD carried out the ansformati rocess according to the referee reports. WH corrected typos and grammatical errors throughout the ma cript, makl/g it more readable. All authors read and approved the final manuscript.

\section{Author details}

${ }^{1}$ College of Resources and Environment, Hunan Agricultural University, Changsha, 4 ? 2 2ollege of Forestry, Central South University of Forestry and Technology, Changsha, 410004, China. ${ }^{3}$ Facult, Science and Technology, Tapee College, Surathani, 84000, Thailand.

\section{Publisher's Note}

Springer Nature remains neutral with regard to jurisdictional cln publisheo, haps and institutional affiliations.

Received: 26 May 2017 Accepted: 27 October 2017 Pab. ed on e: 09 November 2017

\section{References}

1. Gasiorowicz, S: Elementary Particle Physics. Vh N, Yor' (1966)

2. Antosik, P, Mikusinski, J, Sikorski, R: Theor y of Dis tions ne Sequential Approach. PWN-Polish Scientific Publishers, Warsaw (1973)

3. Bremermann, J: Distributions, Cor blex ables, ana Fourier Transforms. Addison-Wesley, Reading (1965)

4. Gelfand, I, Shilov, G: Generalized Eunctions, 1,Academic Press, New York (1964)

5. Bliedtner, J, Hansen, W: Pot ntial Theory: An f, ralytic and Probabilistic Approach to Balayage. Springer, Berlin (1986)

6. Ikegami, T: Compactificati is of Martin type of harmonic spaces. Osaka J. Math. 23, 653-680 (1986)

7. Bliedtner, J, Loeb, P: Best $f$ s for the ceneral Fatou boundary limit theorem. Proc. Am. Math. Soc. 123, 459-463 (1995)

8. Loeb, P: The optin differentiation basis and liftings of $L^{\infty}$. Trans. Am. Math. Soc. 352, 4693-4710 (2000)

9. Samuelsson, H: A re gu.

10. Passare, M. Residues carren,s, and their relation to ideals of holomorphic functions. Math. Scand. 62, 75-152 (1988)

11. Berndt $\mathrm{s}_{\mathrm{N}}$ Passar. M: Integral formulas and an explicit version of the fundamental principle. J. Funct. Anal. 84, $358>(19$ )

12. Andel IVI, Cumsson, $\mathrm{H}$ : $H^{P}$-Estimates of holomorphic division formulas. Pac. J. Math. 173, 307-335 (1996)

12 Dickenst 4 Gay, R, Sessa, C, Yger, A: Analytic functionals annihilated by ideals. Manuscr. Math. 90, 175-223 (1996)

uriol, C, Fel eira, M: Orthogonal decomposition and asymptotic behavior for a linear coupled system of Maxwell and equations. Electron. J. Differ. Equ. 2015, 142 (2015)

15. Aus in Toure, K, Coulibaly, A, Kouassi, AAH: Riesz basis and exponential stability for Euler-Bernoulli beams with variable coefficients and indefinite damping under a force control in position and velocity. Electron. J. Differ. Equ. 2015, 54 (2015)

Yan, Z: Sufficient conditions for non-stability of stochastic differential systems. J. Inequal. Appl. 2015, 377 (2015)

17. Wang, J, Huang, B, Yamini, N: An augmented Riesz decomposition method for sharp estimates of certain boundary value problem. Bound. Value Probl. 2016, 156 (2016) 